\title{
The Glucagon-like Polypeptides - A Reply
}

Dear Sir,

Criticism being infinitely preferable to indifference, I am most grateful to Bloom for his letter and I hope that I can clear up some obvious misunderstandings concerning the original article. Freedom is, like discipline, a highly emotive word with many shades of different meanings. I trust that the correspondant has not ". . . confused the free with the free and easy" with regard to a disciplined approach to a precise and unambiguous terminology.

In "real life", even in the Hammersmith Hospital, the size of an antigenic determinant has a well defined upper limit which is related to the size of the antibody combining site. Studies with antibodies raised against poly-amino acids have indicated that only 5 or 6 residues can be accommodated in this site [1]. As Bloom implies, however, an antigenic determinant is defined by its conformation, as well as by its sequence, so that an antibody directed against a particular site in an intact molecule may react differently with the same site in a fragment of the molecule or when the molecule forms part of a larger sequence. It is not surprising, therefore, that an antibody behaves differently in a radioimmunoassay compared with an immunocytochemical system in which a hormone may be present in a different molecular form or be firmly associated with a cellular component. It is hoped that the development of in vitro techniques of mono-specific antibody production, by Milstein and co-workers [2], will eventually remove some of the sources of confusion which arise from antibody heterogeneity.

The molecular weights of the glucagon-like polypeptides are, indeed, approximations (but, surely, not so far from reality as to be of interest to readers of Hans Christian Andersen) and were included in order to suggest the probable mode of processing of proglucagon by endopeptidases. In addition to this biosynthetic scheme, the glucagon-like polypeptides may be subjected to further post-translational modifications, such as cleavage by exopeptidases, sulphation of tyrosine residue and glycosylation which give rise to the "microheterogeneity" observed in this family of polypeptides.

With regard to measurements in plasma, it was pointed out that the concentration of substances in the plasma which inhibit the binding of radio-labelled glucagon to $\mathrm{N}$-terminally directed antibodies, is of uncertain significance in molecular and physiological terms. The prospect, therefore, of subtracting from this concentration a second concentration of equally doubtful significance to obtain yet a third value fills me with dismay. If the term "plasma enteroglucagon" means precisely the immunoreactivity in plasma measured with $\mathrm{N}$-terminal specific antisera, this writer readily concedes that the term has the virtues of conciseness and simplicity which may be appropriate to certain clinical studies. On the other hand, it seems incongruous to refer to the enteroglucagon content of a tissue other than the gut. The term "Glicentin-like immunoreactivity" is probably not relevant to studies in humans. Glicentin is a polypeptide that was isolated from porcine intestine and whilst the primary structure of glucagon is conserved in all mammalian species except the guinea pig, preliminary results from the Novo group indicate considerable interspecies variation in the sequences of the N-terminal extensions to glucagon. In consequence, antibodies raised against glicentin may only cross-react weakly with glucagon-like polypeptides of human origin [3].

In response to Dr. Bloom's final plea, I would like to remind him of the last words of Madame Roland "O Freedom, what crimes are committed in your name".

Yours faithfully,

\section{J. M. Conlon}

\section{References}

1. Kabat A (1966) The nature of an antigenic determinant. J Immunol 97: 1-11

2. Kohler G, Milstein C (1975) Continuous cultures of fused cells secreting antibody of predefined specificity. Nature 256: 495-497

3. Moody AJ, Sundby F, Jacobsen H, Lauritsen KB (1978) The tissue distribution and plasma levels of glicentin (gut GLI-I). Scand J Gastroenterol 13 (Suppl 49): 127

Dr. J. M. Conlon

Department of Clinical Biochemistry

Royal Victoria Infirmary

Newcastle upon Tyne NE 4LP

England 